\title{
An improved genetic algorithm in path planning for mobile robot Guangrui Liu ${ }^{1, a}$, Xin Tian ${ }^{2, b}$,Wenbo Zhou ${ }^{3, c}$, Kefu Guo ${ }^{4, d}$ Mechanical Engineering school, Zhengzhou University, Zhengzhou Henan 450001, China agrui2006@163.com, b709736128@qq.com , c260397231@qq.com, dguokefu@163.com
}

Keywords: Genetic Algorithm; Path planning; Crossover and mutation; Adaptive adjustment; The elite preservation strategy; Metropolis Guidelines

\begin{abstract}
This paper proposed a new way of crossover and mutation for genetic algorithm to prevent the local optima and guarantee the feasibility of the mutated path. Improving the adaptive adjustment of crossover and mutation probability to improve the search efficiency of the algorithm optimization. Contrary to the disadvantages of genetic algorithm, such as it's easy to fall into the optimal local and premature, the Metropolis based on simulated annealing algorithm is used for the optimization of genetic algorithm. By using the improved genetic algorithm to different environment models and comparing with other genetic algorithms, the results show that the use of improved genetic algorithm has better convergence speed and optimization capabilities.
\end{abstract}

\section{Introduction}

Genetic algorithm is a parallel and global search technology, due to its multi-point calculation in parameter space, so it has more chances to convergence to global optimal, what's more, it does not require the search space differentiable or continuous. Through the global optimization and high concurrency of genetic algorithm, many scholars have tried by using genetic algorithm to various problems of the robot path planning in recent years. References [1] proposed an improved genetic algorithm of path planning method based on a raster map, introducing the path fix to improve the convergence speed of genetic algorithm. References [2] has combined the rough set theory and genetic algorithm together, using rough sets method to simplify the initial population and have the optimization of feasible paths by genetic algorithm, improving the calculation speed greatly. References [3] has robot path planning based on genetic algorithms in discrete space, however, this method is based on static environmental model. References [4] has used the fuzzy control for real-time tuning of crossover and mutation probability, guarantee the quality of path planning.

\section{Path planning based on improved genetic algorithm}

This section has improved on crossover and mutation, the adjustment of adaptive parameter, elite save policy and some other improvements based on genetic algorithm.

Environmental modeling and population coding. A grid-based representation is used for the static environment space of robot motion in our improved GA. The space is a 2-D space and some static obstacles are known by their absolute coordinates. The space is recorded as SG, the number and location of obstacles are known. We establish a rectangular coordinates system, in which the left corner of SG is the origin, the positive direction of y axis is straight down and the level right is the positive direction of X-axis. $X_{\max }$ and $Y_{\max }$ are the maximum range of robot which can move in the $\mathrm{X}$ and $\mathrm{Y}$ directions. In the space, the barrier grid is black and the free grid is white, the width of grid is a unit of length. This paper identifies the grid through the serial number and the rectangular coordinates, the serial number of grid and its corresponding rectangular coordinates are map relationships to each other. The rectangular coordinates of $P(i, j)$ is defined as: 


$$
\left\{\begin{array}{l}
X(i, j)=\bmod (p(j, j), 10)+0.5 \\
Y(i, j)=f \operatorname{loor}(p(i, j) / 10)+0.5
\end{array}\right.
$$

where floor represents rounding down and mod is remainder operator. We use serial number method to express the path while the fitness of it is calculated by the rectangular coordinates. Environmental model and the path expression are shown in Fig. 1.

The environmental model is starting with 0 points, ending with 99 points. Each path, for example, [0 11122233435364757586879 89 99] is an individual of the population.

Population initialization. Traditional genetic algorithm initializes a population randomly, which will produce a large number of infeasible path in path planning, increase the amount of computation, affect the convergence rate. To solve this problem, prior knowledge is used to initialize the population. The steps are as follows:

Step 1: Starting from 0 point, select the right, the down and the lower right probabilities, the next path grid is one of the free adjacent grids. For example, $P(i)$ is the current grid, $P(i)+1$, $P(i)+10, P(i)+11$ are the next adjacent grid in three directions, respectively right, down and the right below;

Step 2: if $P(i+1)$ is not an obstacle gird and is not over spatial boundaries, $P(i+1)$ is the next grid,otherwise, we need get back and choose again;

Step 3: When the number of $p(i)$ is 99 ,we save this individual as a feasible path and regenerates the next individual;

Step 4: When the number of individuals reaches the number of population, the population initialization is completed.

Fitness function. The objective function is generally considered to be the shortest path in path planning, due to the individuals of the population in this paper are viable paths, there is no need to set penalty functions for infeasible paths, in this way we simplify the fitness function and accelerate the running speed. The fitness function is defined as follows:

$$
\text { fitsess }=1 /((1+1 / \sqrt{T-1}) L)
$$

where $T$ is the number of path endpoints, $L$ is the length of path.

Selection method. In this paper, we use roulette wheel selection method to select individuals, the selection which based on the individual fitness.

Crossover method. To ensure the continuous possibility of path, single-point crossover method has been used. As to the random intersections, we do crossover operation only if the two cross points are the same serial number.

After each genetic manipulation, individuals are divided into high fitness group and low fitness group, then randomly select an individual from each of the two groups to crossover, which can make better individual and poorer individual have a greater probability to cross , in this way, the population can have the evolution in an optimal direction and prevent the local optima at the same time. The parent with larger fitness value is compared to the progeny with larger value after crossover, if the progeny is better than the parent,replace the parent with progeny, otherwise,do not replace. In this way, the better one can always be chosen to the next generation, which guarantee the algorithm always has the evolution in the optimal direction.

Mutation. Mutation increases the diversity of the population, expanding the search space of feasible solutions and avoiding premature convergence. The mutation of traditional genetic algorithms is a random mutation, which can produce a infeasible path containing of barrier grid, Fig. 2 shows the infeasible path produced by random mutations. 

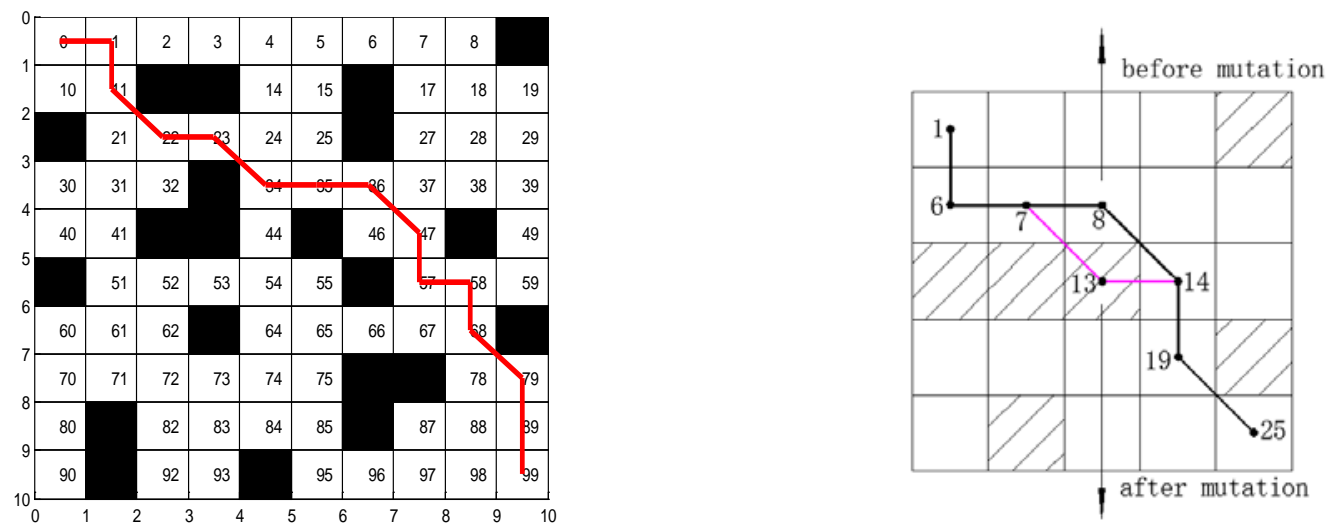

Fig.1 Diagram of the environmental modeling and path. Fig.2 The infeasible path produced by random mutations.

A new mutation is presented as follows:

1) Randomly selected one point $P(j)$ except starting point and destination point from the individual;

2) Have the judgment of the next adjacent grid in the direction of right, down and the lower right.

3) if the adjacent grid is free and is not over spatial boundaries, the mutations of three directions have been done. If the path has the obstacle grid after mutation,delete this path, otherwise, save the path and get to the next step;

4) Calculating the lengths of feasible paths $L 1, L 2, L 3$, the length of original path is $L 4$;

5) Do not change the path if the shortest path is the original one, otherwise, replace the original with the new shortest one.

By using the location search technology and prior knowledge, the mutation improve the search quality and accelerate the convergence.

The transformation process is shown in Fig.3:

1)The original path is [1 6781419 25],the variation node is randomly selected as grid 8;

2)The grid 9,grid 13,grid 14 are free grids and not over spatial boundaries, the mutations of three directions have been done;

3)The path after the right direction mutation is [1 67914 19 25], the down path is [1 671314 19 25], the lower right is [1 $\begin{array}{llll}6 & 7 & 14 & 19\end{array}$ ],three path are available and the length of each one is $L 1, L 2$ 和 $L 3$, the original length is $L 4$;

4) The length L3 is the shortest,replace the original path with new path [1 671419 25].

Adaptive adjustment. Adaptive general algorithm(AGA) was proposed by Srinivas $\mathrm{M}$ and Patnaik L.M in 1994,the crossover and mutation probability can meet the changes of fitness. $f_{\max }$

is the best fitness value in the group, $f_{\text {avg }}$ is the average fitness value of group, we can use

$d=f_{\max }-f_{\text {avg }}$ to express the diversity of the population. The smaller $\mathrm{d}$ is , the lower diversity of the group, the easier the group into local optimums, at this time, we need to increase the crossover and mutation probability to produce new individuals to increase the diversity. On the contrary, when $d$ is increasing, which means the characteristics of individuals are scattered, the crossover and mutation probability need to be decreased at this time. In addition, when the fitness of the individual is higher than the average, the algorithm uses less Pc and Pm, so that the best individuals can be better access to the next generation, while when the fitness of the individual is lower than the average, decrease the Pc and Pm to accelerate the speed of individual updates. The 
crossover $P c$ and mutation probability $\mathrm{Pm}$ can be decided by $f_{\max }-f_{\text {avg }}$ as follows:

$$
\begin{aligned}
& P_{c}= \begin{cases}\frac{k 1\left(f_{\mathrm{m}}-f\right)}{f_{\operatorname{mix}}-f_{\mathrm{sez}}}, & f \geq f_{\mathrm{sez}} \\
k 2 & , f<f_{\mathrm{sez}}\end{cases} \\
& P_{m}= \begin{cases}\frac{k 3\left(f_{\operatorname{mix}}-f^{\prime}\right)}{f_{\operatorname{mix}}-f_{\mathrm{vox}}}, & f^{\prime} \geq f_{\mathrm{vox}} \\
k 4 & , f^{\prime}<f_{\mathrm{vex}}\end{cases}
\end{aligned}
$$

where $f_{\max }$ is the best fitness value in the group; $f_{\text {avg }}$ is the average fitness value of group; $f$ is the larger fitness of the two cross individuals; $f$ ' is the fitness of variation individual; $k 1, k 2, k 3, k 4$ are constant.

The boundaries of early and late lies in the dispersed of individual fitness, that is the size of population diversity, so a criterion is required for calculating the diversity of the population appropriately. Standard deviation is one of the most important criteria for calculating the diversity of observations from the mean value. This is the reason why in this paper, standard deviation of the fitness value of individuals is used as the demarcation criteria of early and late. The standard deviation of the fitness values of individuals is calculated as follows:

$$
\sigma=\sqrt{\frac{1}{n} \sum_{i=1}^{n}\left(f_{i}-f_{m=2}\right)^{2}}
$$

where $\sigma$ is standard deviation of the fitness value of individuals, $\mathrm{n}$ is the number of individuals, $f_{i}$ is the fitness value of $\mathrm{i}$-th individual, and $f_{\text {avg }}$ shows the average of the fitness values. If $\sigma$ is more than $\mathrm{TH}$, the crossover and mutation probability is fixed as the traditional genetic algorithms. If the value of $\sigma$ is less than TH (i.e., threshold value), the diversity of the population is diminished. The value of Pc and Pm must be increased in order to keep the population diversity.

The elite preservation. As a result of using the improved adaptive adjustment, which makes the good individuals involved in genetic manipulation in the early evolution of the populations, in order to make sure that good individuals not be lost, the elite save method has been used:

Step 1: Find the best individual of contemporary species;

Step 2: The best individual is compared with the ever best one, if the best individual is better than the ever best one, this best individual changes to the ever best one;

Step 3: Replace the individual with lowest fitness value with the ever best one.

The metropolis criterion of simulated annealing algorithm. In order to ensure the genetic algorithm escape from the local optimal effectively, the metropolis criterion is used to the receive judgment of the old and new individuals. Transition probability of metropolis criterion is defined as follows:

$$
P(i \rightarrow j)= \begin{cases}1 & , f(j) \leq f(j) \\ e^{\left(\frac{f(j)-f(j)}{t}\right)} & , f(j)>f(i)\end{cases}
$$

$f(i)$ is the fitness value of the current solution and $f(j)$ is value of the new solution. A new solution is generated near the current solution randomly, if $f(j)$ is smaller than $f(i)$,accept the 
new solution as the current solution, or receive the new solution according to the type (6). $t$ is temperature parameter and it has a higher value at the beginning and it can accept the worse deterioration, then $t$ is decreased gradually with the increasing algebra of population, the algorithm can accept better deterioration, at the end of algorithm and $t$ is tending to zero, it can not accept any deterioration. This is why the algorithm can jump out of a local optimum.

The process of improved algorithm is shown as follows:

1)Setting the population size pop size, maximum number of genetic $G$, initial temperature $T=T 0$,annealing factor $q$, and generate the initial population $P($ gen $)$;

2)Define the fitness function fitness and calculate the fitness of each individual $f_{i}$, according to fitness value for the roulette wheel selection;

3)Improved crossover operator;

4)Improved mutation;

5)Use the metropolis criterion to determine whether accept the new individual or not;

6)Elite save policy;

7)Updated the algebra gen=gen +1 , number of temperature $n=n+1$ and the temperature parameter $T n=q^{*} T 0$. If the algebra reach the predetermined number of iteration, finish the algorithm, or turn to the Step 2.

\section{Simulation}

In order to demonstrate the ability of the proposed method in robot motion planning problem, two environments are used for comparison.Fig. 4 shows the result of traditional genetic algorithms simulation, the best path is [11 22334455666778 89 99]. Fig.5 shows the result of improved genetic algorithms simulation, the best path is [11 22334455666778 99]. It can be found that after mutation, the path 79-89-99 changes to 78-99, the path has been optimized and the algorithm is effective. What's more, Fig.6 and Fig.7 are the optimize curve of traditional and improved algorithm. The results shows that the improved method accelerates the speed of convergence to the optimal solution significantly.

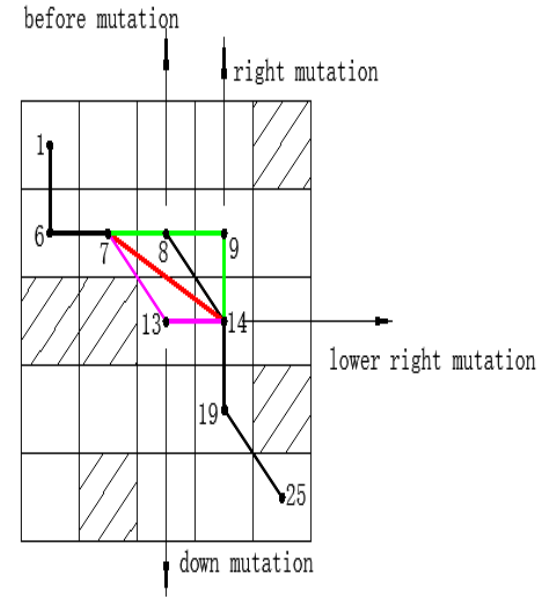

Fig.3Improved mutation schematic.

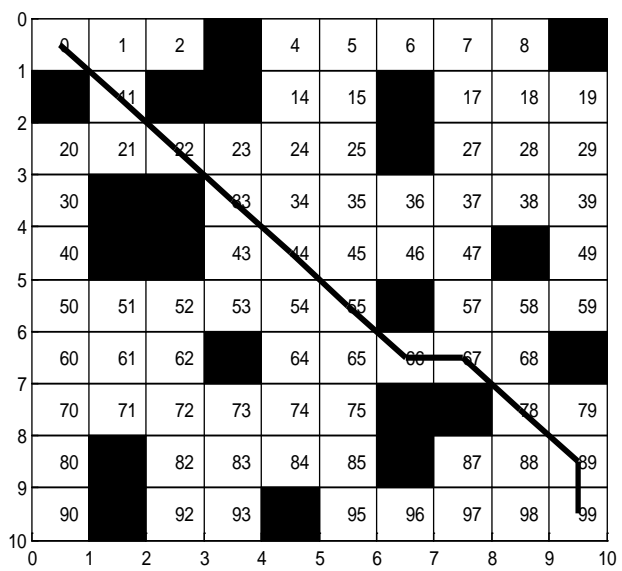

Fig.4Path planning schematics of traditional genetic algorithm. 

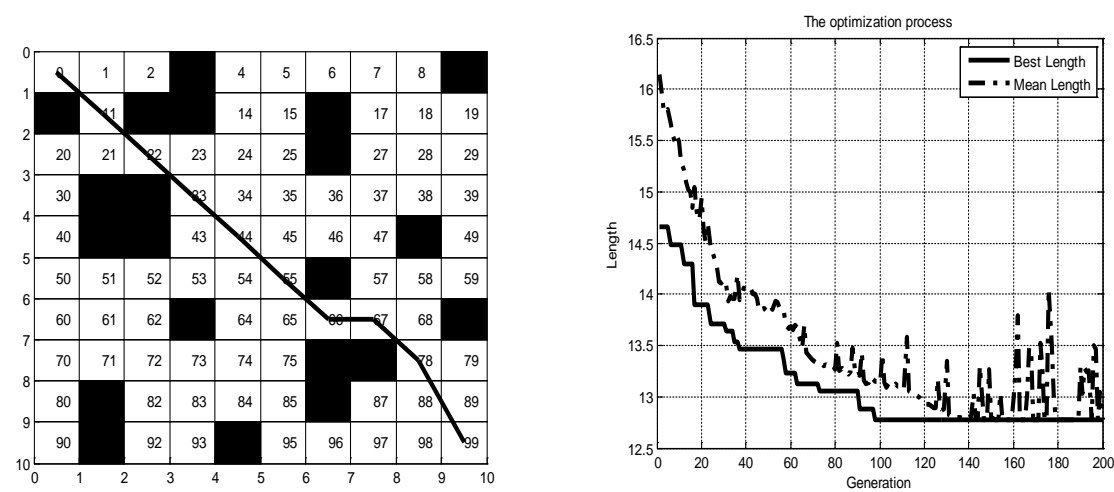

Fig.5Path planning schematics of improved genetic algorithm. Fig.6The optimize curves without elite save policy.
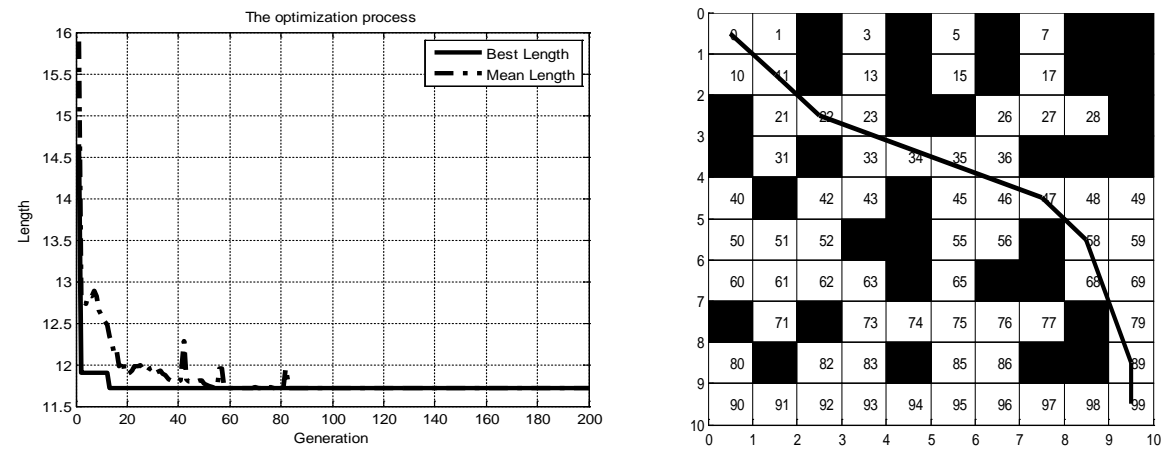

Fig.7. The optimize curves with elite save policy. Fig.8. Path-planning simulation of improved genetic algorithm.

The second environment model is established based on literature [6]. Fig.8 shows the simulation of improved genetic algorithm.

\section{Conclusion}

The results show that the improved genetic algorithm in this paper not only has a higher success rate in searching the best path, but also achieve remarkable results in convergence and path optimization result.

\section{References}

[1] Xu Meijing , Sun Chenliang . Genetic algorithm about route planning based on raster map [J].Science Information , 2011,(31):496-497.

[2] Li Tongtao . Study of robot route planning method based on rough set theory and genetic algorithm . [D]. Zhengzhou : Zhengzhou university ,2007.

[3] Sun Shudong, Qu Yanbin .Application study of genetic algorithm in robot route planning .[N].Journal of northwest industry university , 1998,16(1):79-83.

[4] Li Yajie .Research of intelligent car route planning based on genetic algorithm [D].Liaoning : Liaoning engineering and technology university , 1998,16(1):79-83.

[5] Gao Hanping ,Kang Lishan ,Yang Zuqiao ,Xiao Xiaohong .Evolution algorithm based on self-adaption cross and aberration rate [N].Journal of Huanggang normal college , 2003,23(3):57-60.

[6] Du Zongzong .Study of mobile robot route planning based on genetic algorithm [D].Wuxi :Jiangnan university , 2009. 